\title{
Metabolism and Pharmacokinetic Drug-Drug Interaction Profile of Vericiguat, A Soluble Guanylate Cyclase Stimulator: Results From Preclinical and Phase I Healthy Volunteer Studies
}

\author{
Michael Boettcher ${ }^{1} \cdot$ Michael Gerisch $^{2} \cdot$ Maximilian Lobmeyer $^{1} \cdot$ Nina Besche ${ }^{5} \cdot$ Dirk Thomas $^{3} \cdot$ Mireille Gerrits $^{4}$. \\ Julia Lemmen $^{2} \cdot$ Wolfgang Mueck $^{1} \cdot$ Martin Radtke $^{2} \cdot$ Corina Becker $^{1}$
}

Published online: 26 May 2020

(c) The Author(s) 2020

\begin{abstract}
Background Vericiguat is a stimulator of soluble guanylate cyclase currently under investigation as a first-in-class therapy for worsening chronic heart failure (NCT02861534). Patients with heart failure often require polypharmacy because of comorbidities. Hence, understanding the clearance mechanisms, elimination, and potential for pharmacokinetic drug-drug interactions of vericiguat is important for dose recommendations in this patient population.

Methods Biotransformation and perpetrator properties of vericiguat were characterized in vitro using human hepatocytes, liver microsomes, and recombinant enzymes. This was complemented by a human mass balance study and ten drug-drug interaction studies in healthy volunteers wherein vericiguat was co-administered orally with omeprazole, magnesium/aluminum hydroxide, ketoconazole, rifampicin, mefenamic acid, midazolam, warfarin, digoxin, sacubitril/valsartan, aspirin, or sildenafil. Results In the human mass balance study, mean total radioactivity recovered was $98.3 \%$ of the dose administered (53.1\% and $45.2 \%$ excreted via urine and feces, respectively). The main metabolic pathway of vericiguat is glucuronidation via uridine diphosphate-glucuronosyltransferase 1A9 and 1A1. In vitro studies revealed a low risk of vericiguat acting as a perpetrator by inhibiting cytochrome P450s, uridine diphosphate-glucuronosyltransferase isoforms, or major transport proteins, or by inducing cytochrome P450s. These observations were supported by phase I drug-drug interaction studies. Phase I studies that assessed the propensity of vericiguat as a victim drug showed changes in the range that did not warrant recommendations for dose adjustment in phase III.

Conclusions A low pharmacokinetic interaction potential of vericiguat was estimated from in vitro data and confirmed in vivo. Thus, vericiguat is suitable for a patient population with multiple comorbidities requiring polypharmacy.
\end{abstract}

\section{Key Points}

Michael Boettcher and Michael Gerisch contributed equally to this work.

Electronic supplementary material The online version of this article (https://doi.org/10.1007/s40262-020-00895-x) contains supplementary material, which is available to authorized users.

Corina Becker

corina.becker@bayer.com

1 Clinical Pharmacology, Bayer AG, Aprather Weg 18a, 41113 Wuppertal, Germany

2 DMPK, Bayer AG, Aprather Weg 18a, 41113 Wuppertal, Germany

3 Experimental Medicine, Bayer AG, Aprather Weg 18a, 41113 Wuppertal, Germany
A low pharmacokinetic interaction potential of vericiguat was estimated from in vitro data and confirmed in ten clinical phase I drug-drug interaction studies

The pharmacokinetic drug-drug interaction profile of vericiguat is well suited to the treatment of a heart failure population using multiple concomitant medications

Vericiguat was well tolerated in all studies

4 Merck Sharp \& Dohme Corp., a subsidiary of Merck \& Co., Inc., Kenilworth, NJ, USA

5 Chrestos Concept GmbH \& Co. KG, Girardetstr. 1-5, 45131 Essen, Germany 


\section{Introduction}

Vericiguat is a stimulator of soluble guanylate cyclase (sGC) currently under investigation as a first-in-class therapy for worsening chronic heart failure (HF) with reduced ejection fraction (HFrEF; VICTORIA [NCT02861534]) and preserved ejection fraction (HFpEF; VITALITYHFpEF [NCT03547583]) [1, 2]. Vericiguat was administered orally once daily and titrated from 2.5 up to $10 \mathrm{mg}$ in the phase III VICTORIA study, and is being up-titrated to $15 \mathrm{mg}$ in the phase II VITALITY-HFpEF study. Vericiguat restores the nitric oxide (NO)-sGC-cyclic guanosine monophosphate pathway by directly stimulating sGC independently of $\mathrm{NO}$ and sensitizing sGC to NO, thus restoring cyclic guanosine monophosphate production even under low NO conditions and oxidative stress [1,3]. The first sGC approved for clinical use was riociguat [4], which was developed as a treatment for pulmonary arterial hypertension and chronic thromboembolic pulmonary hypertension $[5,6]$. The clinical benefit profile of riociguat is different to vericiguat [7], and both sGCs have distinct pharmacokinetic (PK) properties.

Patients with HF often present with multiple comorbidities and receive multiple concomitant medications $[8,9]$. In the European Heart Failure Pilot Survey, $74 \%$ of patients with HF experienced one or more comorbidities [10]. Current guideline-recommended therapies propose a stepwise approach to treatment, which frequently involves multiple drugs to treat underlying causative factors, such as hypertension, myocardial ischemia, atrial fibrillation, diabetes mellitus, and dyslipidemia, further increasing the number of prescribed drugs $[8$, 11-13]. Thus, an understanding of the clearance mechanisms, elimination, and potential for drug-drug interactions (DDIs) of vericiguat is important to define allowable co-medications and is a prerequisite for further dose recommendations.

Vericiguat is a low-soluble, highly permeable, and weakly basic Biopharmaceutics Classification System class II drug [14]. Studies in healthy volunteers have demonstrated that vericiguat exhibits linear pharmacokinetics and investigated vericiguat's pharmacodynamic interaction potential $[15,16]$. Higher bioavailability and lower variability of vericiguat after administration with food compared to without has also been reported [14]. Vericiguat is a low-clearance drug $(1.6 \mathrm{~L} / \mathrm{h}$ in healthy volunteers and $1.3 \mathrm{~L} / \mathrm{h}$ in patients with $\mathrm{HFrEF}$ ) [17]. The plasma protein binding of vericiguat is approximately $98 \%$, with serum albumin being the main binding component (data on file). The plasma protein binding is not affected by renal or hepatic impairment. Beyond the titration regimen, no further dose adjustment was recommended for patients with renal impairment (estimated glomerular filtration rate $\geq 15 \mathrm{~mL} / \mathrm{min} / 1.73 \mathrm{~m}^{2}$ ) or for patients with mild-tomoderate hepatic impairment in the VICTORIA study [1].

Following metabolite profiling in human hepatocytes, the primary biotransformation pathway of vericiguat was identified as phase II uridine diphosphate-glucuronosyltransferase (UGT)-mediated conjugation with glucuronic acid (glucuronidation) [18]. Herein, we report results identifying the UGT isoforms contributing to glucuronidation of vericiguat and the contribution of organs to the overall glucuronidation process. Further in vitro studies with human liver microsomes indicate that vericiguat has no inhibitory effects on major cytochrome P450 (CYP) isoforms [18]. Additional preclinical characterization was conducted to identify relevant vericiguat and metabolite M-1 (a pharmacologically inactive major plasma $\mathrm{N}$-glucuronide metabolite) transporter proteins and the inhibitor/inducer properties of vericiguat and M-1 towards various enzymes. In addition, the different excretion pathways of vericiguat in humans and its metabolite profile were investigated in a human mass balance study. Ten healthy volunteer phase I studies further investigated the DDI profile of vericiguat based on its absorption/solubility, metabolism, preclinical findings, as well as with potential concomitant medications.

\section{Methods}

\subsection{In Vitro Studies}

Identification of the UGT isoforms involved in the glucuronidation of vericiguat, as well as transport proteins for vericiguat, was performed by several in vitro studies (see Methods section and Table 1 of the Electronic Supplementary Material [ESM]). In addition, the potential of vericiguat to act as a perpetrator by inhibiting metabolizing enzymes or transporters as well as inducing CYPs was investigated.

\subsection{Phase I Studies}

The conduct of all clinical studies met their local legal and regulatory requirements. All studies were conducted in Germany except for the human mass balance study, which was conducted in the Netherlands. The studies were conducted in accordance with the ethical principles that have their origin in the Declaration of Helsinki and the International Conference on Harmonisation guideline E6: Good Clinical Practice. Informed consent was obtained from all individual participants included in the studies. Quantitative measurements of vericiguat and all other drugs were performed using validated methods and in accordance with relevant guidelines (see Table 2 of the ESM for further details) $[19,20]$. 
Healthy white male individuals were eligible in line with accepted criteria for healthy volunteer studies [21]. Across the studies, the general inclusion criteria for healthy volunteers were (with some variability depending on the study): aged $18-65$ years, body mass index $18-30 \mathrm{~kg} / \mathrm{m}^{2}$, resting heart rate $45-100$ beats per minute, systolic blood pressure 90-145 mmHg, diastolic blood pressure 45-95 mmHg, and an absence of clinically relevant changes in electrocardiogram findings. Participants were excluded if they were taking regular medications, consuming foods or beverages that could influence the study objectives, unable to abide by study-specific smoking restrictions, or taking supplements with known interaction potential.

\subsection{Study Design}

One phase I study investigated the clearance mechanisms and elimination of vericiguat and ten phase I studies investigated the potential for DDIs with vericiguat in healthy male volunteers (Table 1). As vericiguat has been demonstrated to exhibit linear pharmacokinetics [16], the use of different doses of vericiguat across the phase I studies is not considered a limitation. In addition, the investigated doses include the target therapeutic dose range of vericiguat in HF $[1,2]$. While the studies were conducted in relatively small population sizes and in healthy volunteers, all studies were performed to guideline recommendations and designed to investigate specific DDIs. Further details can be viewed in the Methods section of the ESM.

\subsection{Pharmacokinetic and Statistical Analyses}

Pharmacokinetic parameters were estimated using noncompartmental methods by WinNonlin software (Pharsight Corporation, Mountain View, CA, USA). Area under the plasma concentration-time curve (AUC) from 0 to infinity was calculated from the AUC from time 0 to the last quantifiable concentration using the linear-logarithmic trapezoidal method and from the last predicted plasma concentration and apparent terminal rate constant (calculated from the slope of a log-linear regression). The linear-logarithmic trapezoidal method was also used to calculate partial AUCs (e.g., $\mathrm{AUC}_{0-24}$ [AUC from time 0 to $24 \mathrm{~h}$ after administration]) and the AUC within the dosing interval after multiple dosing $\left(\mathrm{AUC}_{\tau, \mathrm{md}}\right)$. The maximum concentration $\left(C_{\max }\right)$ and the trough concentration within a dosing interval were directly determined from the concentration-time data for each participant. Summary descriptive statistics were provided for the cumulative excretion of total radioactivity in urine, feces, and urine plus feces in the human mass balance study.

For the DDI studies, PK parameters with and without comedications were compared. Depending on the design of the study, selected PK parameters were analyzed if applicable and assumed to be log-normally distributed. These included AUC, $\mathrm{AUC}_{0-24}, \mathrm{AUC}_{0-24, \mathrm{md}}\left(\mathrm{AUC}_{0-24}\right.$ after multiple-dose administration), $\mathrm{AUC}_{0-22}$ (AUC from time 0 to $22 \mathrm{~h}$ ), $\mathrm{AUC}_{0-12, \text { md }}$ (AUC from time 0 to $12 \mathrm{~h}$ after multiple-dose administration), $\mathrm{AUC}_{\tau, \mathrm{md}}, C_{\max }, C_{\text {max,md }}\left(C_{\max }\right.$ within a dosing interval after multiple-dose administration), and trough concentration within a dosing interval. The logarithms of these parameters were analyzed using analysis of variance, including sequence, healthy volunteer (sequence), period, and treatment effects, except for the rifampicin, sacubitril/ valsartan, and sildenafil DDI studies, which only included healthy volunteer and treatment effects. Based on these analyses, point estimates (least-squares mean) of the geometric mean ratios and $90 \%$ confidence intervals for the treatment ratios were calculated by retransformation of the logarithmic data.

\subsection{Safety and Tolerability}

Adverse events, vital signs, electrocardiogram, and laboratory parameters (i.e., hematology, coagulation, clinical chemistry, and urinalysis) in relation to the study drug were captured for all phase I studies.

\section{Results}

\subsection{In Vitro Studies}

In vitro studies showed that glucuronidation of vericiguat was catalyzed primarily by UGT1A9 and UGT1A1. Human liver, kidney, and intestinal microsomes fortified with uridine 5'-diphospho-glucuronic acid mediate glucuronidation of vericiguat. In human liver microsomes of single donors $(n=20)$, the formation of M-1 correlated slightly better with propofol glucuronidation $\left(r^{2}=0.73\right)$, a highly selective UGT1A9-mediated pathway, than with estradiol 3 -glucuronidation $\left(r^{2}=0.61\right)$, a highly selective UGT1A1mediated pathway. Niflumic acid, a potent UGT1A9 inhibitor, showed stronger inhibition on vericiguat glucuronidation than a potent UGT1A1 inhibitor (atazanavir), suggesting that glucuronidation of vericiguat could be preferentially catalyzed via UGT1A9 than via UGT1A1. Further results are presented in Tables 3 and 4 of the ESM.

Additional in vitro studies have determined that vericiguat is a substrate of the transporter proteins P-glycoprotein and breast cancer resistance protein. Vericiguat is not a substrate of organic anion transporting polypeptide (OATP) 1B1, OATP1B3, or organic cation transporter 1. Metabolite M-1 is not a substrate of efflux (P-glycoprotein, breast cancer resistance protein) or uptake transporters (OATP1B1, OATP1B3). The design and interpretation of the in vitro studies were in line with official guidance [22,23]. 


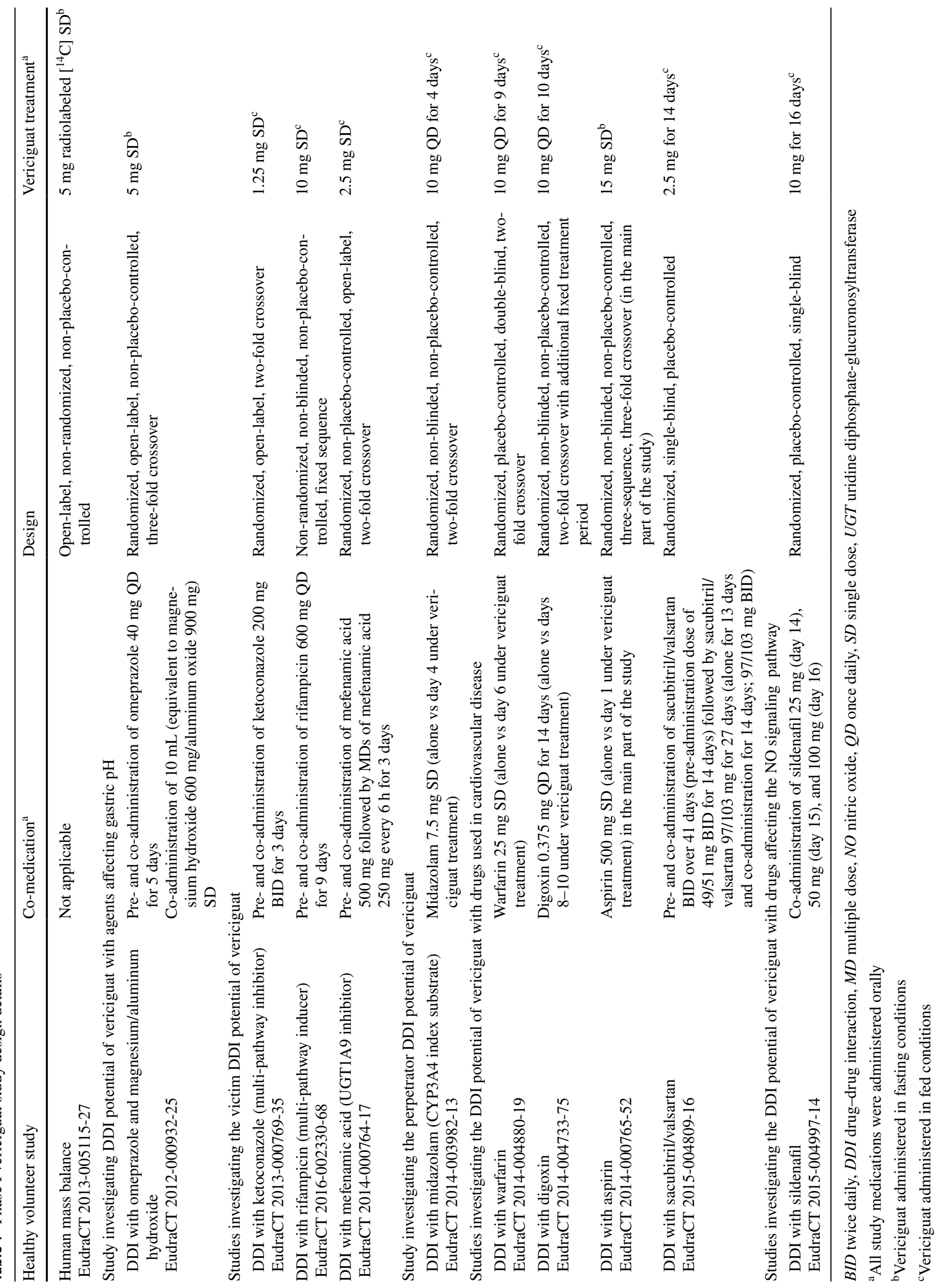


Table 2 Demographics of healthy volunteers (pharmacokinetic analysis set) in six phase I studies investigating the clearance mechanisms, elimination, and potential for drug-drug interactions (DDIs) of vericiguat as a victim or perpetrator

\begin{tabular}{lllllll}
\hline Characteristic & $\begin{array}{l}\text { Human mass balance } \\
(n=6)\end{array}$ & $\begin{array}{l}\text { DDI with omeprazole } \\
\text { and magnesium/alu- } \\
\text { minum hydroxide } \\
(n=10)\end{array}$ & $\begin{array}{l}\text { DDI with } \\
\text { ketocona- } \\
\text { zole } \\
(n=14)\end{array}$ & $\begin{array}{l}\text { DDI with rifampicin } \\
(n=15)\end{array}$ & $\begin{array}{l}\text { DDI with } \\
\text { mefenamic } \\
\text { acid } \\
(n=13)\end{array}$ & $\begin{array}{l}\text { DDI with midazolam } \\
(n=32)\end{array}$ \\
\hline $\begin{array}{l}\text { Male, } n(\%) \\
\text { White, } n(\%)\end{array}$ & $6(100)$ & $10(100)$ & $14(100)$ & $15(100)$ & $13(100)$ & $32(100)$ \\
$\begin{array}{l}\text { Age range } \\
\text { criterion, years } \\
\text { (inclusive) }\end{array}$ & $45-65$ & $10(100)$ & $14(100)$ & $15(100)$ & $13(100)$ & $32(100)$ \\
$\begin{array}{l}\text { Weight range, } \mathrm{kg} \\
\text { Height range, } \mathrm{cm}\end{array}$ & $60.3-102.4$ & $18-45$ & $18-45$ & $18-45$ & $18-55$ & $18-55$ \\
BMI range, $\mathrm{kg} / \mathrm{m}^{2}$ & $19.1-29.0$ & $75.0-88.0$ & & & $65-95$ \\
\hline
\end{tabular}

Values are range (minimum-maximum) unless stated otherwise

$B M I$ body mass index

Furthermore, no inhibition was observed for vericiguat and its metabolite (M-1) at the highest tested concentration for efflux transporters $P$-glycoprotein and bile salt export pump (half maximal inhibitory concentration $\left.\left[\mathrm{IC}_{50}\right]>100 \mu \mathrm{M}\right)$, multidrug and toxin extrusion protein (MATE) 1 and MATE2K $\left(\mathrm{IC}_{50}>10 \mu \mathrm{M}\right)$, as well as for uptake of organic cation transporter $1\left(\mathrm{IC}_{50}>50 \mu \mathrm{M}\right)$, organic anion transporter (OAT) 1, OAT3, and organic cation transporter $2\left(\right.$ all $\left.\mathrm{IC}_{50}>5 \mu \mathrm{M}\right)$. Both vericiguat and M-1 did not inhibit major UGT isoforms (UGT1A1, 1A4, 1A6, 2B4, and 2B7; all $\mathrm{IC}_{50}>50 \mu \mathrm{M}$ ), except for UGT1A9 inhibition by vericiguat $\left(\mathrm{IC}_{50}=10.6 \mu \mathrm{M}\right.$, inhibitory constant $\left.\left[\mathrm{K}_{\mathrm{i}}\right]=6.6 \mu \mathrm{M}\right)$. In addition, vericiguat is an inhibitor of breast cancer resistance protein $\left(\mathrm{IC}_{50}=20 \mu \mathrm{M}\right)$ as well as OATP1B1 and 1B3 (approximate $\mathrm{IC}_{50}$ of 16 and $30 \mu \mathrm{M}$, respectively). Metabolite M-1 is an inhibitor of OATP1B1 and OATP1B3 $\left(\mathrm{IC}_{50}=25.6\right.$ and $16.6 \mu \mathrm{M}$, respectively). However, considering the clinical exposure of vericiguat (total $C_{\max }$ approximately $0.8 \mu \mathrm{M}$, unbound $C_{\max }$ approximately $0.02 \mu \mathrm{M}$, and projected unbound inlet $\mathrm{C}_{\max } 0.03 \mu \mathrm{M}$; data on file) and M-1 (total $C_{\max } 2.7 \mu \mathrm{M}$ and unbound $C_{\max } 0.04 \mu \mathrm{M}$; data on file) following a $10-\mathrm{mg}$ dose once daily, the DDI risk assessment according to the US Food and Drug Administration and European Medicines Agency guidelines indicated a low potential for vericiguat and M-1 to cause DDIs when co-administered with substrates of major CYP and UGT isoforms as well as transporters.

The CYP induction potential of vericiguat and M-1 was evaluated in cultured human hepatocytes from three different donors. Vericiguat showed no significant induction of CYP1A2 and CYP2B6 up to the highest tested concentration of $10,000 \mu \mathrm{g} / \mathrm{L}(23.5 \mu \mathrm{M})$. For CYP3A4, a 2.7-fold increase in CYP3A4 messenger RNA was observed in one of the three donors at the highest tested concentration (4\% of the rifampicin [positive control] response). The potential risk for
CYP3A4 induction in the clinic was further assessed using the relative induction score method according to the US Food and Drug Administration and European Medicines Agency DDI guidance $[22,23]$. The in vitro induction data in human hepatocytes (CYP3A4 messenger RNA data) and the clinical exposure data from multiple-dose oral administration of $10 \mathrm{mg}$ of vericiguat once daily in patients with HFrEF (total $C_{\max }$ approximately $1 \mu \mathrm{M}$ and unbound $C_{\max }$ approximately $0.02 \mu \mathrm{M}$; data on file) were used as input data. The relative induction score model predicted a $15 \%$ decrease in the AUC of midazolam, indicating that vericiguat is likely not a clinically meaningful inducer of CYP3A4 in vivo.

\subsection{Demographics of Healthy Volunteers in all Phase I Studies}

The characteristics of the healthy volunteers enrolled in each of the studies who were valid for PK analyses are shown in Tables 2, 3. Studies were conducted between 2012 and 2017.

\subsection{Human Mass Balance Study}

A total of six healthy volunteers received a single oral solution containing ${ }^{14} \mathrm{C}$-labeled vericiguat $5 \mathrm{mg}$. Mean total radioactivity (range) recovered in urine and feces within $336 \mathrm{~h}$ was $98.3 \%$ (95.6-99.8\%) of the dose administered. Of the administered dose, $53.1 \%(49.9-56.1 \%)$ and $45.2 \%$ (40.1-49.9\%; mean values [range]) were excreted via urine and feces, respectively.

$N$-glucuronide metabolite M-1, a pharmacologically inactive major plasma metabolite with no known off-target activities, represented the majority of the radioactivity in urine (Fig. 1, Fig. 1 of the ESM, and Table 4; $40.8 \%$ of the dose) together with vericiguat ( $9.0 \%$ of the dose). Metabolite M-15, resulting from oxidative metabolism (presumably by 
Table 3 Demographics of healthy volunteers (pharmacokinetic analysis set) in five phase I studies investigating the potential for drug-drug interactions (DDIs) of vericiguat with drugs prescribed in cardiovascular disease or acting on the nitric oxide signaling pathway

\begin{tabular}{|c|c|c|c|c|c|c|c|}
\hline \multirow[t]{2}{*}{ Characteristic } & \multirow{2}{*}{$\begin{array}{l}\text { DDI with } \\
\text { warfarin } \\
(n=23)\end{array}$} & \multirow{2}{*}{$\begin{array}{l}\text { DDI with digoxin } \\
(n=25)\end{array}$} & \multirow{2}{*}{$\begin{array}{l}\text { DDI with } \\
\text { aspirin } \\
\text { (main part) } \\
(n=14)\end{array}$} & \multicolumn{2}{|c|}{ DDI with sacubitril/valsartan } & \multicolumn{2}{|c|}{ DDI with sildenafil } \\
\hline & & & & $\left(n=15 ; \mathrm{PKS} 1^{\mathrm{a}}\right)$ & $\left(n=14 ; \mathrm{PKS}^{\mathrm{b}}\right)$ & $\left(n=16 ; \mathrm{PKS}^{\mathrm{a}}\right)$ & $\left(n=32 ; \mathrm{PKS}^{\mathrm{b}}\right)$ \\
\hline Male, $n(\%)$ & $23(100)$ & $25(100)$ & $14(100)$ & $15(100)$ & $14(100)$ & $16(100)$ & $32(100)$ \\
\hline White, $n(\%)$ & $23(100)$ & $25(100)$ & $14(100)$ & $15(100)$ & $14(100)$ & $16(100)$ & $32(100)$ \\
\hline $\begin{array}{l}\text { Age range } \\
\text { criterion, years } \\
\text { (inclusive) }\end{array}$ & $18-55$ & $18-55$ & $18-45$ & $40-60$ & $40-60$ & $40-60$ & $40-60$ \\
\hline Weight range, kg & $62.1-98.2$ & $54.3-95.5$ & $63-93$ & $67.3-96.0$ & $67.3-96.0$ & $61.4-93.5$ & $61.4-101.4$ \\
\hline Height range, $\mathrm{cm}$ & $164-188$ & $162-189$ & $163-186$ & $165.0-186.0$ & $165.0-186.0$ & $168.0-188.0$ & $168.0-191.0$ \\
\hline BMI range, $\mathrm{kg} / \mathrm{m}^{2}$ & $20.5-29.0$ & $20.1-29.8$ & $21.6-28.7$ & $21.5-29.2$ & $21.5-28.1$ & $21.4-29.1$ & $21.2-29.5$ \\
\hline
\end{tabular}

Values are range (minimum-maximum) unless stated otherwise

$B M I$ body mass index, $P K S$ pharmacokinetic set

${ }^{\text {a }}$ Population used for the analysis of the victim potential of vericiguat with co-medication

${ }^{\mathrm{b}}$ Population used for the analysis of the perpetrator potential of vericiguat with co-medication

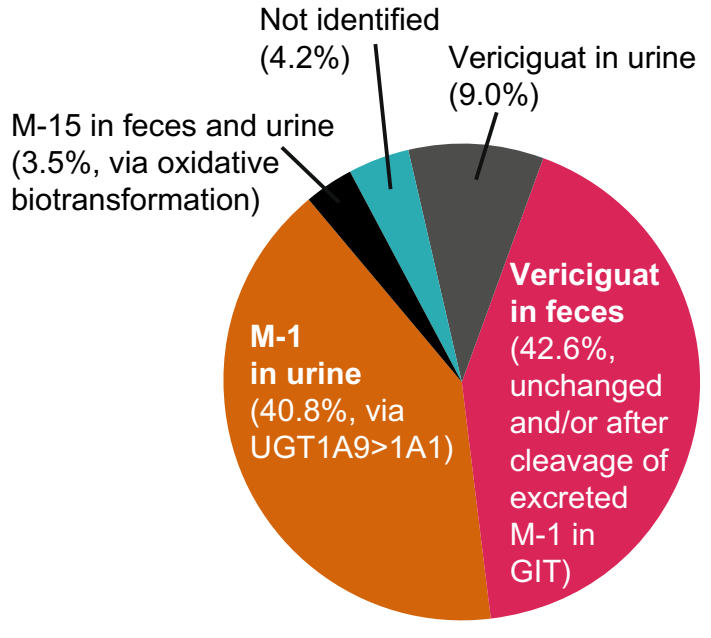

Fig. 1 Excretion and clearance of vericiguat. GIT gastrointestinal tract, $U G T$ uridine diphosphate-glucuronosyltransferase demethylation/decarboxylation/acetylation), was detected as a minor component in urine and feces $(1.9 \%$ and $1.6 \%$, respectively). Vericiguat represented most of the radioactivity in feces (42.6\% of the dose). Metabolite M-1 was the predominant component in human plasma, accounting for $72 \%$ of total radioactivity AUC, compared with $27 \%$ for the parent drug.

\subsection{Drug-Drug Interaction Studies}

The clinical interaction studies confirmed and extended findings from the in vitro studies (Fig. 2, Tables 5 and 6, and Tables 5 and 6 of the ESM).

\subsubsection{Effects of Drugs Affecting Intestinal pH on the Pharmacokinetics of Vericiguat}

Pre- and co-administration of omeprazole with vericiguat reduced the AUC and $C_{\max }$ of vericiguat by $32 \%$ and $49 \%$,
Table 4 Excretion pattern of vericiguat and its metabolites ${ }^{\mathrm{a}}$

\begin{tabular}{llll}
\hline Analyte & Total (range), $\%$ & $\begin{array}{l}\text { Excretion in feces } \\
\text { (range), } \%\end{array}$ & Excretion in urine (range), \% \\
\hline Vericiguat & $51.6(46.0-62.6)$ & $42.6(38.3-46.5)$ & $9.0(6.1-16.1)$ \\
M-1 & $40.8(30.3-47.4)$ & - & $40.8(30.3-47.4)$ \\
M-15 & $3.5(2.1-4.8)$ & $1.6(0.3-2.5)$ & $1.9(1.6-2.3)$ \\
Other & $1.9(0.8-4.1)$ & $1.0(0.4-2.7)$ & $0.9(0.1-1.5)$ \\
Total recovery & $98.3^{\mathrm{b}}(95.6-99.8)$ & $45.2(40.1-49.9)$ & $53.1^{\mathrm{b}}(49.9-56.1)$ \\
\hline
\end{tabular}

${ }^{\mathrm{a}}$ Results from the human mass balance study

${ }^{\mathrm{b}} \mathrm{A}$ difference ( $0.5 \%$ of dose) to the sum of vericiguat, M-1, M-15, and others resulted from not investigated urine samples at later timepoints due to low radioactivity 


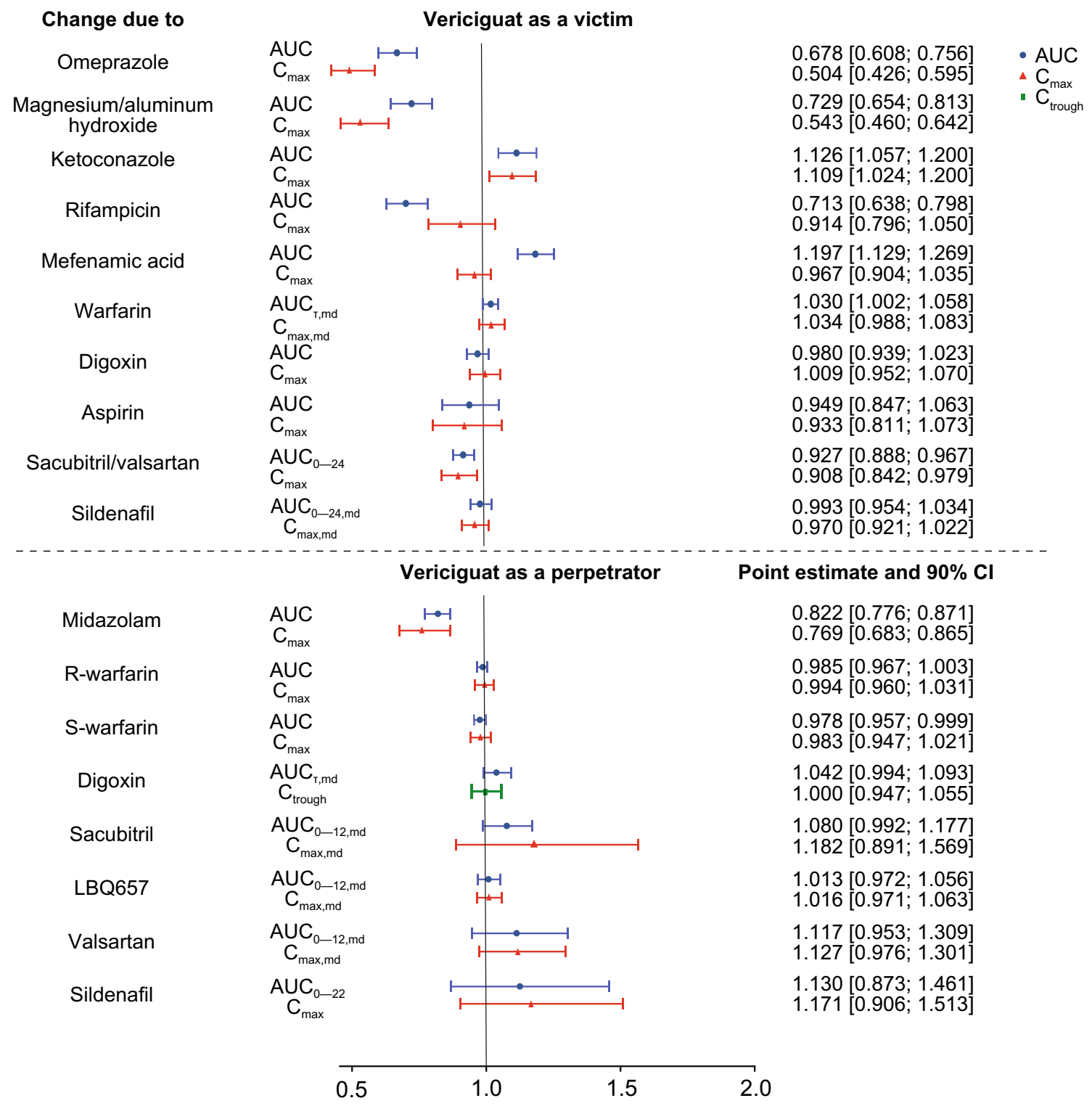

Fig. 2 Summary of clinical interactions and pharmacokinetic changes investigating the perpetrator and victim potential of vericiguat. $A U C$ area under the concentration-time curve, $A U C_{0-12, m d}$ AUC from time 0 to $12 \mathrm{~h}$ after multiple dose administration, $A U C_{(0-22)}$ AUC from time 0 to $22 \mathrm{~h}, A U C_{(0-24)}$ AUC from time 0 to $24 \mathrm{~h}, A U C_{(0-24), m d}$

respectively, compared with administration of vericiguat alone. Similar results were observed with co-administration of magnesium/aluminum hydroxide with vericiguat (Table 5).

\subsubsection{Effects of Drugs on the Pharmacokinetics of Vericiguat}

Co-administration with ketoconazole (a multi-pathway CYP and transporter inhibitor) increased vericiguat exposure
$\mathrm{AUC}_{0-24}$ after multiple-dose administration, $A U C_{\tau, m d}$ AUC within the dosing interval after multiple dosing, $C I$ confidence interval, $C_{\max }$ maximum concentration within a dosing interval, $C_{\max , m d} \mathrm{C}_{\max }$ within a dosing interval after multiple dose administration, $C_{\text {trough }}$ trough concentration within a dosing interval to a minor extent (by 13\%). Pre- and co-treatment with rifampicin (multi-pathway UGT, CYP, and transporter inducer) led to a decrease in the AUC and $C_{\max }$ of vericiguat by $29 \%$ and $9 \%$, respectively. Inhibition of UGT1A9 by mefenamic acid led to a non-relevant increase in the AUC of vericiguat by $20 \%$ (Table 5).

Changes in the pharmacokinetics of vericiguat when given in combination with drugs prescribed in cardiovascular disease or those affecting the NO signaling pathway compared with no co-administration are also shown in Table 5. No 
Table 5 Effects of other drugs on the pharmacokinetics of vericiguat

\begin{tabular}{|c|c|c|c|c|c|}
\hline \multirow[t]{2}{*}{ Interaction drug } & \multicolumn{5}{|c|}{ Changes in the PK parameters of vericiguat } \\
\hline & PK parameter & $N$ & Point estimate & $\begin{array}{l}90 \% \text { confidence } \\
\text { interval }\end{array}$ & $\begin{array}{l}\text { Clinical comment in relation to the VICTORIA } \\
\text { study }\end{array}$ \\
\hline \multirow[t]{2}{*}{ Omeprazole } & AUC & 10 & 0.678 & $0.608-0.756$ & \multirow{2}{*}{$\begin{array}{l}\text { No dose adjustment of vericiguat when given } \\
\text { with a pH-modifying agent }\end{array}$} \\
\hline & $C_{\max }$ & 10 & 0.504 & $0.426-0.595$ & \\
\hline \multirow[t]{2}{*}{ Magnesium/aluminum hydroxide } & AUC & 10 & 0.729 & $0.654-0.813$ & \multirow{2}{*}{$\begin{array}{l}\text { No dose adjustment of vericiguat when given } \\
\text { with a pH-modifying agent }\end{array}$} \\
\hline & $C_{\max }$ & 10 & 0.543 & $0.460-0.642$ & \\
\hline \multirow[t]{2}{*}{ Ketoconazole } & AUC & 15 & 1.126 & $1.057-1.200$ & \multirow{2}{*}{$\begin{array}{l}\text { No dose adjustment of vericiguat when given } \\
\text { with a multi-pathway inhibitor }\end{array}$} \\
\hline & $C_{\max }$ & 15 & 1.109 & $1.024-1.200$ & \\
\hline \multirow[t]{2}{*}{ Rifampicin } & AUC & 15 & 0.713 & $0.638-0.798$ & \multirow{2}{*}{$\begin{array}{l}\text { No dose adjustment of vericiguat when given } \\
\text { with a multi-pathway inducer }\end{array}$} \\
\hline & $C_{\max }$ & 15 & 0.914 & $0.796-1.050$ & \\
\hline \multirow[t]{2}{*}{ Mefenamic acid } & AUC & 13 & 1.197 & $1.129-1.269$ & \multirow{2}{*}{$\begin{array}{l}\text { No dose adjustment of vericiguat when given } \\
\text { with a UGT1A9 inhibitor }\end{array}$} \\
\hline & $C_{\max }$ & 13 & 0.967 & $0.904-1.035$ & \\
\hline \multicolumn{6}{|c|}{ In combination with drugs prescribed in cardiovascular disease } \\
\hline \multirow[t]{2}{*}{ Warfarin } & $\mathrm{AUC}_{\tau, \mathrm{md}}$ & 23 & 1.030 & $1.002-1.058$ & \multirow[t]{2}{*}{ No dose adjustment of vericiguat } \\
\hline & $C_{\text {max,md }}$ & 23 & 1.034 & $0.988-1.083$ & \\
\hline \multirow[t]{2}{*}{ Digoxin } & AUC & 24 & 0.980 & $0.939-1.023$ & \multirow[t]{2}{*}{ No dose adjustment of vericiguat } \\
\hline & $C_{\max }$ & 24 & 1.009 & $0.952-1.070$ & \\
\hline \multirow[t]{2}{*}{ Aspirin } & AUC & 14 & 0.949 & $0.847-1.063$ & \multirow[t]{2}{*}{ No dose adjustment of vericiguat } \\
\hline & $C_{\max }$ & 14 & 0.933 & $0.811-1.073$ & \\
\hline \multirow[t]{2}{*}{ Sacubitril/valsartan } & $\mathrm{AUC}_{0-24}$ & 15 & 0.927 & $0.888-0.967$ & \multirow[t]{2}{*}{ No dose adjustment of vericiguat } \\
\hline & $C_{\max }$ & 15 & 0.908 & $0.842-0.979$ & \\
\hline \multicolumn{6}{|c|}{ In combination with drugs affecting the NO signaling pathway } \\
\hline \multirow[t]{2}{*}{ Sildenafil $^{\mathrm{a}}$} & $\mathrm{AUC}_{0-24, \mathrm{md}}$ & 14 & 0.993 & $0.954-1.034$ & \multirow{2}{*}{$\begin{array}{l}\text { Excluded from the phase III VICTORIA study } \\
\text { as patient safety data is not available }\end{array}$} \\
\hline & $C_{\text {max,md }}$ & 14 & 0.970 & $0.921-1.022$ & \\
\hline
\end{tabular}

$A U C$ area under the concentration-time curve, $A U C_{(0-24)} \mathrm{AUC}$ from time 0 to $24 \mathrm{~h}, A U C_{(0-24), m d} \mathrm{AUC}_{0-24}$ after multiple-dose administration, $A U C_{\tau, m d}$ AUC within the dosing interval after multiple dosing, $C_{\max }$ maximum concentration within a dosing interval, $C_{\text {max }, m d} \mathrm{C}_{\max }$ within a dosing interval after multiple-dose administration, $C Y P$ cytochrome $\mathrm{P} 450, D D I$ drug-drug interaction, $N O$ nitric oxide, $P K$ pharmacokinetic, $U G T$ uridine diphosphate-glucuronosyltransferase

${ }^{a}$ Data for the highest dose of sildenafil administered (100 mg)

differences between the PK parameters of vericiguat when given alone in comparison with its co-administration with warfarin, digoxin, or aspirin were observed. The analysis of variance results for the $\mathrm{AUC}_{0-24}$ and $\mathrm{C}_{\max }$ showed decreases in the exposure of vericiguat of about $7 \%$ and $9 \%$, respectively, when vericiguat was administered with sacubitril/ valsartan. While the $90 \%$ confidence intervals for both parameters excluded unity, the magnitude of change in exposure was small and not considered clinically relevant. Sildenafil co-administration at different doses had no relevant effect on vericiguat $\mathrm{PK}$ parameters. Of note, vericiguat exposure was decreased following co-administration with sildenafil $50 \mathrm{mg}$, but the differences in these PK parameters were small (less than 9\%), did not increase at a higher sildenafil dose $(100 \mathrm{mg})$, and hence were considered irrelevant. A mean difference in maximum decrease in seated blood pressure of $\leq 4-5 \mathrm{mmHg}$ between multiple doses of vericiguat $10 \mathrm{mg}$ with single doses of sildenafil $(25,50$, or $100 \mathrm{mg})$ and placebo with single doses of sildenafil $(25,50$, or $100 \mathrm{mg})$ was observed. No dose-dependent trend was observed with the different sildenafil doses.

\subsubsection{Effects of Vericiguat on the Pharmacokinetics of Other Drugs}

A slight reduction in midazolam exposure (by 18\%) was observed after 3 days pre-treatment and 1 day co-treatment with vericiguat $10 \mathrm{mg}$ (Table 6). The AUC and $C_{\max }$ of 1 '-hydroxy midazolam, the main metabolite of midazolam, also decreased by $12 \%$ and $16 \%$, respectively. It is noted that the molecular weight-adjusted metabolite-to-parent ratio was comparable ( 0.27 after administration of midazolam alone vs 0.29 after co-administration of midazolam with vericiguat), suggesting no effect on CYP3A4-mediated metabolism by vericiguat.

Vericiguat did not alter the pharmacokinetics of R- and $\mathrm{S}$-warfarin or digoxin with point estimates for the treatment ratios (co-medication plus vericiguat/co-medication) of AUC and $C_{\max }$ near 1. Vericiguat at steady state resulted in 
Table 6 Effects of vericiguat on the pharmacokinetics of other drugs

\begin{tabular}{|c|c|c|c|c|c|}
\hline \multirow[t]{2}{*}{ Interaction drug } & \multicolumn{5}{|c|}{ Changes in the PK parameters of interaction drugs } \\
\hline & PK parameter & $N$ & Point estimate & $\begin{array}{l}90 \% \text { confidence } \\
\text { interval }\end{array}$ & $\begin{array}{l}\text { Clinical comment in relation to the VICTORIA } \\
\text { study }\end{array}$ \\
\hline \multirow[t]{2}{*}{ Midazolam } & AUC & 32 & 0.822 & $0.776-0.871$ & \multirow{2}{*}{$\begin{array}{l}\text { No dose adjustment of co-medications metabo- } \\
\text { lized by CYP3A4 }\end{array}$} \\
\hline & $C_{\max }$ & 32 & 0.769 & $0.683-0.865$ & \\
\hline \multicolumn{6}{|c|}{ In combination with drugs prescribed in cardiovascular disease } \\
\hline \multirow[t]{2}{*}{ R-warfarin } & AUC & 23 & 0.985 & $0.967-1.003$ & \multirow[t]{2}{*}{ No dose adjustment of warfarin } \\
\hline & $C_{\max }$ & 23 & 0.994 & $0.960-1.031$ & \\
\hline \multirow[t]{2}{*}{ S-warfarin } & AUC & 23 & 0.978 & $0.957-0.999$ & \multirow[t]{2}{*}{ No dose adjustment of warfarin } \\
\hline & $C_{\max }$ & 23 & 0.983 & $0.947-1.021$ & \\
\hline \multirow[t]{2}{*}{ Digoxin } & $\mathrm{AUC}_{\tau, \mathrm{md}}$ & 22 & 1.042 & $0.994-1.093$ & \multirow[t]{2}{*}{ No dose adjustment of digoxin } \\
\hline & $C_{\text {trough }}$ & 22 & 1.000 & $0.947-1.055$ & \\
\hline \multirow[t]{2}{*}{ Sacubitril } & $\mathrm{AUC}_{0-12, \mathrm{md}}$ & 14 & 1.080 & $0.992-1.177$ & \multirow[t]{2}{*}{ No dose adjustment of sacubitril/valsartan } \\
\hline & $C_{\text {max,md }}$ & 14 & 1.182 & $0.891-1.569$ & \\
\hline \multirow[t]{2}{*}{ LBQ657 } & $\mathrm{AUC}_{0-12, \mathrm{md}}$ & 14 & 1.013 & $0.972-1.056$ & \multirow[t]{2}{*}{ No dose adjustment of sacubitril/valsartan } \\
\hline & $\mathrm{C}_{\max , \mathrm{md}}$ & 14 & 1.016 & $0.971-1.063$ & \\
\hline \multirow[t]{2}{*}{ Valsartan } & $\mathrm{AUC}_{0-12, \mathrm{md}}$ & 14 & 1.117 & $0.953-1.309$ & \multirow[t]{2}{*}{ No dose adjustment of sacubitril/valsartan } \\
\hline & $C_{\text {max,md }}$ & 14 & 1.127 & $0.976-1.301$ & \\
\hline \multicolumn{6}{|c|}{ In combination with drugs affecting the NO signaling pathway } \\
\hline \multirow[t]{2}{*}{ Sildenafil $^{\mathrm{a}}$} & $\mathrm{AUC}_{0-22}$ & 30 & 1.130 & $0.873-1.461$ & \multirow{2}{*}{$\begin{array}{l}\text { Currently excluded from the phase III VICTORIA } \\
\text { study as patient safety data is not available }\end{array}$} \\
\hline & $C_{\max }$ & 30 & 1.171 & $0.906-1.513$ & \\
\hline
\end{tabular}

$A U C$ area under the concentration-time curve, $A U C_{0-12, m d}$ AUC from time 0 to $12 \mathrm{~h}$ after multiple dose administration, $A U C_{(0-22)}$ AUC from time 0 to $22 \mathrm{~h}, A U C_{\tau, m d}$ AUC within the dosing interval after multiple dosing, $C_{\max }$ maximum concentration within a dosing interval, $C_{\max , m d}$ $\mathrm{C}_{\max }$ within a dosing interval after multiple dose administration, $C_{\text {trough }}$ trough concentration within a dosing interval, $C Y P$ cytochrome $\mathrm{P} 450$, $D D I$ drug-drug interaction, $N O$ nitric oxide, $P K$ pharmacokinetic

${ }^{\mathrm{a}}$ Data for the highest dose of sildenafil administered $(100 \mathrm{mg})$

increases in the $\mathrm{AUC}_{0-12, \mathrm{md}}$ and $C_{\text {max,md }}$ of sacubitril by $8 \%$ and $18 \%$, respectively, and the $\mathrm{AUC}_{0-12 \text {,md }}$ and $C_{\text {max,md }}$ of valsartan by $12 \%$ and $13 \%$, respectively. Exposure and peak concentration of the active sacubitril metabolite (LBQ657) were unchanged by vericiguat. Co-administration of vericiguat increased exposure and maximum concentration of sildenafil $100 \mathrm{mg}$ with vericiguat in comparison with coadministration of sildenafil with placebo by $13 \%$ and $17 \%$, respectively (Table 6).

\subsection{Safety and Tolerability}

Vericiguat was well tolerated in all studies, and most treatment-emergent adverse events were of mild-to-moderate severity and as expected from the mechanism of action of vericiguat. A total of seven treatment-emergent adverse event-related discontinuations were reported across the DDI studies; however, only one (moderate headache) was related to treatment with vericiguat in the sildenafil DDI study. The treatment-emergent adverse event of moderate headache was considered to be related to both vericiguat and sildenafil.

\section{Discussion}

These in vitro experiments and clinical pharmacology studies characterized the biotransformation, elimination, and DDI potential of vericiguat.

\subsection{Biotransformation and Elimination of Vericiguat}

The glucuronidation of vericiguat is mediated by UGT1A1 and UGT1A9. The preclinical biotransformation experiments demonstrated the dominant role of UGT1A9. Considering the different abundances of these UGT isoforms in tissues, UGT1A9 is predominantly responsible for M-1 formation in the kidneys, whereas both UGT1A9 and UGT1A1 are involved in the liver [24, 25]. In the human mass balance study, M-1 represented the majority of the radioactivity in urine ( $41 \%$ of the dose), while vericiguat represented the majority of the radioactivity in feces $(43 \%$ of the dose). Given the high oral bioavailability (93\%; with food) [14], the unchanged drug recovered in feces is not due to non-absorption. Metabolite M-1 was not detected in human feces; ex vivo incubation with human feces under anaerobic conditions showed that M-1 could undergo hydrolytic cleavage to form vericiguat by microbial flora (Fig. 2 
of the ESM). Based on a preclinical mass balance study in bile-duct cannulated rats, metabolite M-1 can be excreted via bile (data on file). Therefore, the parent drug observed in feces could result from biliary/intestinal secretion of the parent, as well as possible hydrolysis of M-1 in the gut lumen. Thus, although $41 \%$ of the total vericiguat dose is found in the urine as M-1, glucuronidation may contribute to a greater percentage of the overall clearance.

\subsection{Pharmacokinetic Drug-Drug Interaction Potential of Vericiguat}

Ten phase I DDI studies in healthy volunteers investigated the PK interactions between vericiguat and drugs that are known to affect: intestinal $\mathrm{pH}$ and $\mathrm{NO}$ signaling; inhibit/ induce metabolic pathways; and drugs that are commonly administered to patients with cardiovascular disease. Polypharmacy is common in patients with HF and therefore drugs intended to be used in HF are required to possess a low potential for DDIs as these may result in uncontrolled changes in drug plasma concentrations [11-13].

Previous studies have shown an increase in AUC and $\mathrm{C}_{\max }$ by approximately $44 \%$ and $41 \%$, respectively, and lower variability (geometric coefficient of variation in AUC was 19\% vs $41 \%$ ) of vericiguat (alone) after administration with food compared to without $[14,16]$. Here, a reduction by about $30 \%$ in the bioavailability of vericiguat was observed following co-administration in fasting conditions of omeprazole (40 mg once daily) and magnesium/aluminum hydroxide $(600 / 900 \mathrm{mg})$ relative to vericiguat alone. This is primarily driven by the increase in gastric $\mathrm{pH}$, as vericiguat is a basic compound with a $\mathrm{p} K_{\mathrm{a}}$ of 4.7 and its solubility is lower in neutral conditions compared with acidic conditions. The increased exposure following administration with food is believed to be due to enhanced wettability and solubility of vericiguat by stimulation of bile salt secretion and the presence of food components enabling better solubilization. Therefore, vericiguat is recommended to be taken with food, irrespective of food type and the concomitant administration of proton pump inhibitors, as well as antacids, were allowed in phase II and III studies.

No relevant PK interaction was observed with the strong CYP3A and broad-spectrum transporter inhibitor ketoconazole (200 mg twice daily; vericiguat AUC increased by $13 \%)$ or the inducer rifampicin $(600 \mathrm{mg}$ once daily; vericiguat AUC decreased by $29 \%$ ). Therefore, similar concomitant medications were not excluded in the clinical studies. In addition, inhibition of the major metabolizing enzyme of vericiguat, UGT1A9, by mefenamic acid (500 mg four times daily) led to a non-relevant increase in the AUC of vericiguat (by 20\%), thereby allowing UGT inhibitors as concomitant medications in further clinical studies.
The lack of a DDI with midazolam (7.5 mg, single dose) in the clinic is consistent with the in vitro data indicating that vericiguat is not a CYP3A4 inhibitor [18]. Moreover, results from the relative induction score model indicate that vericiguat is likely not a clinically meaningful inducer of CYP3A4 in vivo.

Consistent with the preclinical data, vericiguat had no effect on the pharmacokinetics of narrow therapeutic index drugs commonly prescribed in cardiovascular disease (warfarin $25 \mathrm{mg}$ and digoxin $0.375 \mathrm{mg}$ ). Similarly, warfarin, digoxin, aspirin (500 mg; single dose), and sacubitril/ valsartan (co-administered at $97 / 103 \mathrm{mg}$ twice daily) had no effect on the pharmacokinetics of vericiguat.

To rule out that a potential pharmacodynamic interaction is driven by an underlying PK interaction in the sacubitril/ valsartan interaction study with vericiguat, the plasma concentrations of both compounds were measured. Exposure and peak concentration of the active sacubitril metabolite LBQ657 were unaffected by vericiguat. For valsartan, increases in exposure and peak concentration were similar following co-administration with vericiguat and co-administration with placebo. Therefore, a PK interaction between vericiguat and sacubitril/valsartan was ruled out. Furthermore, multiple oral doses of vericiguat $2.5 \mathrm{mg}$ administered $2 \mathrm{~h}$ prior to sacubitril/valsartan had no additional effect on seated blood pressure [15].

The sildenafil DDI study indicated an increase in exposure for sildenafil co-administered with vericiguat of $22.4 \%$ or less, compared with sildenafil co-administered with placebo; however, all 90\% confidence intervals included one. No meaningful change in the pharmacokinetics of vericiguat in response to sildenafil treatment was observed. No PK dose-dependent trend was observed with sildenafil $(25,50$, or $100 \mathrm{mg}$ ).

Vericiguat was generally well tolerated and had a favorable safety profile across all phase I studies when given for a short duration or as a single dose in healthy volunteers. Most treatment-emergent adverse events were mild or moderate in severity and in line with those previously reported for vericiguat $[26,27]$. However, as the studies were performed in healthy volunteers, the findings may not reflect how the combination of vericiguat and other co-medications will be tolerated in patients. The concomitant use of vericiguat and phospodiesterase- 5 inhibitors, such as sildenafil, is not recommended because of the lack of clinical experience in patients with HF and the potential increased risk for symptomatic hypotension.

Overall, this series of DDI studies demonstrates that vericiguat has a low potential for PK DDIs across the intended therapeutic dose range. Vericiguat has predictable pharmacokinetics, which can be described by a one-compartment model parameterized by apparent clearance and apparent volume of distribution [17]. Based on this population PK 
model from patients with HFrEF [17], variability in steadystate AUC is estimated to be about $30 \%$. Therefore, the observed maximum changes in exposure in the reported studies were within the range of reported PK vericiguat variability in patients with HFrEF and are considered not clinically relevant. The concurrent or anticipated use of drugs affecting gastric $\mathrm{pH}$ and known metabolic pathways, as well as drugs with a narrow therapeutic index or commonly prescribed in patients with cardiovascular disease were permitted in the phase III VICTORIA study.

\section{Conclusions}

A low PK interaction profile was estimated from in vitro studies and confirmed in healthy volunteer studies; changes in vericiguat exposure were in the range of $30 \%$ or less in the phase I DDI studies. Given the low potential for PK interactions, no dose adjustment beyond the dose titration algorithm as per the protocol was required in the phase III VICTORIA study [1]. These results indicate that vericiguat is suitable for the treatment of a patient population with multiple comorbidities requiring polypharmacy, such as patients with HF.

Acknowledgments The authors thank the participants and all investigators involved in this study. Part of this analysis was presented at the 2019 European Society of Cardiology Heart Failure Congress and the 2018 Pharmacokinetics/Pharmacodynamics (PK/PD) Expert Meeting. The authors acknowledge Martina Blombach for technical assistance in analyzing the human mass balance, Armin Kern for investigations on cytochrome P450 induction, and Michael Niehues for partial analysis of in vitro data, all of Bayer AG, Berlin, Germany. Medical writing support, including assisting authors with the development of the outline and initial draft and incorporation of comments was provided by Moamen Hammad, $\mathrm{PhD}$, and editorial support, including fact checking, referencing, figure preparation, formatting, proofreading, and submission was provided by Annabel Ola, MSc, of Scion, London, supported by Bayer AG, Berlin, Germany and Merck Sharp \& Dohme Corp., a subsidiary of Merck \& Co., Inc., Kenilworth, NJ, USA according to Good Publication Practice guidelines. The sponsor was involved in the study design, collection, analysis, and interpretation of data, as well as data checking of information provided in the article. However, ultimate responsibility for opinions, conclusions, and data interpretation lies with the authors.

Author Contributions $\mathrm{MB}, \mathrm{MG}, \mathrm{DT}, \mathrm{MG}, \mathrm{WM}, \mathrm{MR}$, and CB contributed to the conception, design, or planning of the study. MB, MG, JL, and $\mathrm{CB}$ contributed to the acquisition of the data. MB, MG, ML, NB, MG, WM, MR, and CB contributed to the analysis and/or interpretation of data. All authors drafted the article and/or revised it critically for important intellectual content and provided final approval of the published version.

Data Availability Availability of the data underlying this publication will be determined according to Bayer's commitment to the EFPIA/ PhRMA "Principles for responsible clinical trial data sharing". This pertains to scope, timepoint, and process of data access. As such, Bayer commits to sharing upon request from qualified scientific and medical researchers patient-level clinical trial data, study-level clinical trial data, and protocols from clinical trials in patients for medicines and indications approved in the USA and European Union as necessary for conducting legitimate research. This applies to data on new medicines and indications that have been approved by the European Union and US regulatory agencies on or after 1 January, 2014. Interested researchers can use http://www.clinicalstudydatarequest.com to request access to anonymized patient-level data and supporting documents from clinical studies to conduct further research that can help advance medical science or improve patient care. Information on the Bayer criteria for listing studies and other relevant information is provided in the study sponsors section of the portal. Data access will be granted to anonymized patient-level data, protocols, and clinical study reports after approval by an independent scientific review panel. Bayer is not involved in the decisions made by the independent review panel. Bayer will take all necessary measures to ensure that patient privacy is safeguarded.

\section{Compliance with Ethical Standards}

Funding Funding for this research was provided by Bayer AG, Berlin, Germany and Merck Sharp \& Dohme Corp., a subsidiary of Merck \& Co., Inc., Kenilworth, NJ, USA.

Conflict of Interest Maximilian Lobmeyer, Michael Gerisch, Michael Boettcher, Dirk Thomas, Julia Lemmen, Wolfgang Mueck, Martin Radtke, and Corina Becker are employees of Bayer and may own stock in the company. Mireille Gerrits is a former employee of Merck Sharp \& Dohme Corp., a subsidiary of Merck \& Co., Inc., Kenilworth, NJ, USA. Nina Besche is an employee of Chrestos Concept GmbH \& Co. $\mathrm{KG}$, which received funding for this analysis from Bayer AG.

Consent to Participate Informed consent was obtained from all individual participants included in the studies.

Ethics Approval All procedures performed in studies involving human participants were in accordance with the ethical standards of the relevant independent ethics committee or institutional review board and with the 1964 Helsinki Declaration and its later amendments or comparable ethical standards, and the International Conference on Harmonisation Guideline E6: Good Clinical Practice.

Open Access This article is licensed under a Creative Commons Attribution-NonCommercial 4.0 International License, which permits any non-commercial use, sharing, adaptation, distribution and reproduction in any medium or format, as long as you give appropriate credit to the original author(s) and the source, provide a link to the Creative Commons licence, and indicate if changes were made. The images or other third party material in this article are included in the article's Creative Commons licence, unless indicated otherwise in a credit line to the material. If material is not included in the article's Creative Commons licence and your intended use is not permitted by statutory regulation or exceeds the permitted use, you will need to obtain permission directly from the copyright holder.To view a copy of this licence, visit http://creativecommons.org/licenses/by-nc/4.0/.

\section{References}

1. Armstrong PW, Roessig L, Patel MJ, Anstrom KJ, Butler J, Voors AA, et al. A multicenter, randomized, double-blind, placebo-controlled trial of the efficacy and safety of the oral soluble 
guanylate cyclase stimulator: the VICTORIA trial. JACC Heart Fail. 2018;6(2):96-104.

2. Butler J, Lam CSP, Anstrom KJ, Ezekowitz J, Hernandez AF, O'Connor CM, et al. Rationale and design of the VITALITYHFpEF trial. Circ Heart Fail. 2019;12(5):e005998.

3. Armitage ME, Wingler K, Schmidt HH, La M. Translating the oxidative stress hypothesis into the clinic: NOX versus NOS. J Mol Med. 2009;87(11):1071-6.

4. US Food and Drug Administration. Adempas prescribing information. 2017. https://www.accessdata.fda.gov/drugsatfda_docs/label /2017/204819s006lbl.pdf. Accessed 10 Mar 2020.

5. Ghofrani HA, D’Armini AM, Grimminger F, Hoeper MM, Jansa P, Kim NH, et al. Riociguat for the treatment of chronic thromboembolic pulmonary hypertension. N Engl J Med. 2013;369(4):319-29.

6. Ghofrani HA, Galie N, Grimminger F, Grunig E, Humbert M, Jing ZC, et al. Riociguat for the treatment of pulmonary arterial hypertension. N Engl J Med. 2013;369(4):330-40.

7. Frey R, Becker C, Saleh S, Unger S, van der Mey D, Mück W. Clinical pharmacokinetic and pharmacodynamic profile of riociguat. Clin Pharmacokinet. 2018;57(6):647-61.

8. Ponikowski P, Voors AA, Anker SD, Bueno H, Cleland JG, Coats AJ, et al. 2016 ESC guidelines for the diagnosis and treatment of acute and chronic heart failure: the Task Force for the diagnosis and treatment of acute and chronic heart failure of the European Society of Cardiology (ESC) Developed with the special contribution of the Heart Failure Association (HFA) of the ESC. Eur J Heart Fail. 2016;18(8):891-975.

9. Yancy CW, Jessup M, Bozkurt B, Butler J, Casey DE Jr, Colvin MM, et al. 2017 ACC/AHA/HFSA focused update of the 2013 ACCF/AHA guideline for the management of heart failure: a report of the American College of Cardiology/American Heart Association Task Force on Clinical Practice Guidelines and the Heart Failure Society of America. Circulation. 2017;136(6):e137-61.

10. van Deursen VM, Urso R, Laroche C, Damman K, Dahlstrom U, Tavazzi L, et al. Co-morbidities in patients with heart failure: an analysis of the European Heart Failure Pilot Survey. Eur J Heart Fail. 2014;16(1):103-11.

11. Mastromarino V, Casenghi M, Testa M, Gabriele E, Coluccia $\mathrm{R}$, Rubattu S, et al. Polypharmacy in heart failure patients. Curr Heart Failure Rep. 2014;11(2):212-9.

12. McMurray JJ, Pfeffer MA. Heart failure. Lancet. 2005;365(9474):1877-89.

13. Volpe M, Pignatelli G, Paneni F. Polytherapy in cardiovascular prevention: open issues. G Ital Cardiol (Rome). 2012;13(7-8):503-10.

14. Becker C, Boettcher M, Loewen S, Mueck W. Biopharmaceutical profile, bioavailability, food effect, and pharmacokinetics of vericiguat: a novel soluble guanylate cyclase stimulator. American Association of Pharmaceutical Scientists (AAPS); 3-6 November 2019; San Antonio (TX): abstract M1430-13-86. Clinical Pharmacology: Biomolecular. https://showcase.dropbox.com/s/AAPS2019-Conference-Proceedings.-ULgLg54QNJ7BNITbgnmIL.

15. Boettcher M, Loewen S, Gerrits M, Becker C. Pharmacodynamic and pharmacokinetic interaction profile of vericiguat. Eur J Heart Fail. 2019;21(Suppl. S1):294-5.
16. Boettcher M-F, Thomas D, Mueck W, Loewen S, Arens E, Yoshikawa $\mathrm{K}$, et al. Safety, pharmacodynamic and pharmacokinetic characterisation of vericiguat: key results from six phase I studies in healthy subjects. Eur J Heart Fail. 2019;21(Suppl. S1):293.

17. Meyer M, Ruehs H, Klein D, Frei M, Garman D, Grevel J, et al. Population pharmacokinetics and pharmacodynamics of vericiguat in heart failure patients with reduced ejection fraction. Eur J Heart Fail. 2019;21:376.

18. Follmann M, Ackerstaff J, Redlich G, Wunder F, Lang D, Kern A, et al. Discovery of the soluble guanylate cyclase stimulator vericiguat (BAY 1021189) for the treatment of chronic heart failure. J Med Chem. 2017;60(12):5146-61.

19. European Medicines Agency. Guideline on bioanalytical method validation. 2011. https://www.ema.europa.eu/en/documents/scien tific-guideline/guideline-bioanalytical-method-validation_en.pdf. Accessed 3 Jul 2019.

20. US Food and Drug Administration. Guidance for industry: bioanalytical method validation. Rockville, MD, USA: US Department of Health and Human Services, FDA, Center for Drug Evaluation and Research; 2001. https://wayback.archive-it. org/7993/20170111172148/http://www.fda.gov/downloads/ Drugs/GuidanceComplianceRegulatoryInformation/Guidances/ UCM070107.pdf.

21. Breithaupt-Groegler K, Coch C, Coenen M, Donath F, Erb-Zohar $\mathrm{K}$, Francke K, et al. Who is a 'healthy subject'? Consensus results on pivotal eligibility criteria for clinical trials. Eur J Clin Pharmacol. 2017;73(4):409-16.

22. European Medicines Agency. Guideline on the investigation of drug interactions. 2012. https://www.ema.europa.eu/en/docum ents/scientific-guideline/guideline-investigation-drug-interactio ns_en.pdf. Accessed 8 Jul 2019.

23. US Food and Drug Administration. In vitro metabolism and transporter mediated drug-drug interaction studies guidance for industry. 2017. Available from: https://www.fda.gov/media/10813 0/download. Accessed 8 Jul 2019.

24. Bhatt DK, Mehrotra A, Gaedigk A, Chapa R, Basit A, Zhang $\mathrm{H}$, et al. Age- and genotype-dependent variability in the protein abundance and activity of six major uridine diphosphateglucuronosyltransferases in human liver. Clin Pharmacol Ther. 2019;105(1):131-41.

25. Margaillan G, Rouleau M, Fallon JK, Caron P, Villeneuve L, Turcotte V, et al. Quantitative profiling of human renal UDPglucuronosyltransferases and glucuronidation activity: a comparison of normal and tumoral kidney tissues. Drug Metab Dispos. 2015;43(4):611-9.

26. Gheorghiade M, Greene SJ, Butler J, Filippatos G, Lam CS, Maggioni AP, et al. Effect of vericiguat, a soluble guanylate cyclase stimulator, on natriuretic peptide levels in patients with worsening chronic heart failure and reduced ejection fraction: the SOCRATES-REDUCED randomized trial. JAMA. 2015;314(21):2251-62.

27. Pieske B, Maggioni AP, Lam CSP, Pieske-Kraigher E, Filippatos $\mathrm{G}$, Butler J, et al. Vericiguat in patients with worsening chronic heart failure and preserved ejection fraction: results of the SOluble guanylate Cyclase stimulatoR in heArT failurE patientS with PRESERVED EF (SOCRATES-PRESERVED) study. Eur Heart J. 2017;38(15):1119-27. 